\title{
Finding True Change Point When a CUSUM Control Chart is Used
}

\author{
Mohammad Esmaeil Dehghan Monfared* and Fazlollah Lak \\ Persian Gulf University \\ Received: 12/8/2017 Approved: 11/14/2018
}

\begin{abstract}
In this paper, it is assumed that the mean of a normal process is monitored by a CUSUM control chart. When the control chart triggers a signal and declares that the process has gone out of control, a search process is started to find the time of change and the causes of going the process out of control. Several methods (plans) for finding the true (real) change point is proposed. It is shown that the plans which are based on the likelihood of the points in time perform better.
\end{abstract}

Keywords. MLE; change point; CUSUM chart.

MSC 2010: 62F10; 62F15; 62F99.

\section{Introduction}

The quality of products is always the key factor for a successful business. In order to have a high quality of products, the maintenance of a high quality process is essential. In most situations, it is assumed that the quality characteristic of a product follows a normal distribution. The typical technique for maintaining or monitoring a process is the use of statistical process control (SPC) charts. In this article, we will consider the cumulative sum (CUSUM) control chart, which may be used when we are interested in detecting small shifts in the process mean.

\footnotetext{
* Corresponding author

Copyright $(\mathrm{C} 2019$, ASP Ins. This open-access article is published under the terms of the Creative Commons AttributionNonCommercial 4.0 International License which permits Share (copy and redistribute the material in any medium or format) and Adapt (remix, transform, and build upon the material) under the Attribution-NonCommercial terms.
} 
The CUSUM control chart plots the statistics $C_{t}^{+}=\max \left\{0, z_{t}-l+C_{t-1}^{+}\right\}$and $C_{t}^{-}=\max \left\{0,-z_{t}-l+C_{t-1}^{-}\right\}$where the starting values are $C_{0}^{+}=C_{0}^{-}=0$, $z_{t}=\frac{\left(\bar{X}_{t}-\mu_{0}\right)}{\sigma_{0} / \sqrt{n}}$ is the standardized subgroup average, $\sigma_{0}$ is the standard deviation of the process when it is in- control and $l$ is the reference value (almost always taken as 0.5). If the test statistic $C_{t}^{+}\left(C_{t}^{-}\right)$exceeds its decision interval, $h^{+}\left(h^{-}\right)$, one concludes that the process mean has increased (decreased) and that the process is therefore out of control. In this paper we consider a CUSUM chart with reference value $l=0.5$ and decision interval $h^{+}=h^{-}=4.77$ (see Montgomery, 2009). The CUSUM chart with these parameters has an in-control Average Run Length (ARL) of 370, the same as the 3 sigma Shewhart $\bar{X}$ control chart.

It is noteworthy to distinguish the difference between the change point and the out-of-control signal time which is triggered by the SPC charts. The change point is the time that the disturbances affect the process and the SPC signal time is the time that the out-of-control signal will be triggered. In fact, the former is followed by the latter.

Once the control chart signals that the process is out-of-control, the process engineers must initiate a search for the special cause of the process disturbance. The typical method (TM) is to search for the root causes starting from the initial signal time. If the root causes cannot be identified at the initial signal time $T$; the searching process may proceed at time $T-1$. This process should continue until the identification is made. Note that each one of the points $\mathrm{T}, \mathrm{T}-1, \ldots, 1$ can be a possible change point but only one of them is the real change point. In othe words, each one of these points is a false change point except one, which we refer to as the true change point. To find the true change point, some authors have proposed that first, the change point is estimated, usually by maximum likelihood method, and then starting from this estimate of the change point, the search procedure is started to find the true change point and the main causes of going the production process out of control. We call this method the ChronologicalOrdered Method (COM). Note that the TM is also a special case of COM where the change point is estimated by the signal time of the control chart. In their paper, Samuel et al. (1998) addressed the issue of estimating the change point of a normal process. Pignatiello and Samuel (2001) used the EWMA and CUSUM charts and the MLE to estimate the change point of a process. Shao and Hou (2004) provided some statistical properties for the change point estimators. In addition, Shao and Hou (2006) derived the change point estimators under the case where the S chart and MLE are 
used in a gamma process. Later Shao et al (2006) used an X-bar control chart and MLE to estimate the change point of a gamma process. Also, in Machine Learning (ML) approach for the change point problems can be found in Cheng and Cheng (2008) and Shao and Hsu (2009). Shao and Hou (2013a) applied an integrated approach of neural network and analysis of variance to identify a change point in an industrial process. Shao and Hou (2013b) used a two- stage hybrid scheme to estimate a change point for a multivariate process. Hou et al (2013) used a combined MLE and Generalized $\mathrm{P}$ chart approach to estimate the change point of a multinomial process. From Bayesian point of view, Dehghan Monfared and Meshkani (2007) estimated multiple change points in a gamma process when an $\bar{X}$ control chart is used, Dehghan Monfared and Lak (2013) used an X-bar control chart for estimating the change point of a normal process. But the main goal of estimating the change point is to find the true change point and the estimate of the change point is not useful by itself. Dehghan Monfared and Lak (2016) consider the problem from Bayesian perspective. In this paper, we discuss the problem of finding the true change point from classical point of view, when a CUSUM control chart is used. To do this, after the CUSUM control chart triggers a signal, an intuitive method is to sort points in time based on their likelihood and examine the production process at each of those points respectively. Note that, In this case, the starting point is also an estimate, i.e. MLE, of the change point. This method is called likelihood ordered method (LOM) (see Dehghan Monfared and Lak, 2017). For estimating the change point, using maximum likelihood method, most authors have used a conditional likelihood given the signal time T. Dehghan Monfared and Lak (2013) proposed that an unconditional likelihood should be used because the signal time $\mathrm{T}$ has some information about the change point. In this paper, after a CUSUM triggers a signal, LOM and COM are used for each one of two types of likelihood (Note that TM does not depend on the type of the likelihood function which is used) and their performances are compared through a series of simulations.

The paper is organized as follows. In Section 2, the model is defined. In Section 3, an approximation for the distribution of the signal is obtained. In Section 4, two MLEs for the change point are derived. In Section 5, two plans for finding the true change point are introduced. In Section 6 , the methods of finding the true change point are compared through a series of simulations. The paper is concluded in Section 7. 


\section{The Model}

In this study, we assume that the process is initially in control, and the sample observations come from a normal distribution with a known mean, $\mu_{0}$ and a known standard deviation, $\sigma_{0}$. However, after an unknown point in time $\tau$, a disturbance is introduced into the process and starting from the point in time $\tau+1$ (known as the process change point) it changes the process mean from $\mu_{0}$ to $\mu_{1}=\mu_{0}+\delta \sigma_{0} / \sqrt{n}$, where $n$ is the subgroup sample size and $\delta$ is the unknown magnitude of the change in the scale of the standard deviation of the subgroup sample mean (note that $\delta=\frac{\left(\mu_{1}-\mu_{0}\right)}{\sigma / \sqrt{n}}$ ). It is also assumed that once the parameter $\mu_{0}$ changed, it remains at the new level of $\mu_{1}$ until the root causes of the disturbance have been identified and removed. Let $X_{i j}$ denote the $j$ th observation in subgroup $i$ with normal distribution $\mathrm{N}(\cdot, \cdot)$. That is,

$$
\begin{aligned}
X_{i j} \stackrel{i i d}{\sim} N\left(\mu_{0}, \sigma_{0}^{2}\right), \quad i & =1,2, \ldots, \tau \\
j & =1,2, \ldots, n,
\end{aligned}
$$

and

$$
\begin{aligned}
X_{i j} \stackrel{i i d}{\sim} N\left(\mu_{1}, \sigma_{0}^{2}\right), \quad i & =\tau+1, \ldots, T \\
j & =1,2, \ldots, n,
\end{aligned}
$$

where $n$ is the subgroup sample size, $T$ is the signal time that the CUSUM control chart triggers a signal and it is not a false alarm. The parameters $\mu_{0}, \sigma_{0}$ and $\mu_{1}$ are the process parameters, and $\stackrel{i i d}{\sim}$ stands for independent and identically distributed. Note that $T$ is also a random variable, but finding its distribution is not an easy task. In the next section we try to find a good approximation for it.

\section{An Approximation for the Distribution of the Signal Time}

Given that the mean of the process has been changed to $\mu_{1}$ at an unknown time $\tau+1$, since the process is under control until time $\tau$, the probability 
that a signal time is triggered by CUSUM control chart $k$ points in time after $\tau$ is,

$$
\begin{aligned}
h\left(k ; \mu_{1}\right)= & P_{\mu_{1}}\left(T^{\star}=k\right)=P_{\mu_{1}}\left(\left\{C_{\tau+1}^{+}<h^{+}, C_{\tau+1}^{-}<h^{-}\right\} \cap \ldots\right. \\
& \left.\cap\left\{C_{\tau+k-1}^{+}<h^{+}, C_{\tau+k-1}^{-}<h^{-}\right\} \cap\left\{C_{\tau+k}^{+}>h^{+} \text {or } C_{\tau+k}^{-}>h^{-}\right\}\right),
\end{aligned}
$$

where $T^{\star}=T-\tau$. In the last expression $C_{\tau+1}^{+}$and $C_{\tau+1}^{-}$are functions of $C_{\tau}^{+}$and $C_{\tau}^{-}$which are unknown and can be set to be zero because the process is in control for $t \leqslant \tau$. In addition the random variables $C_{\tau+1}^{+}\left(C_{\tau+1}^{-}\right)$, $\cdots, C_{\tau+k}^{+}\left(C_{\tau+k}^{-}\right)$are just computed from a sequence of subgroups sampled from $N\left(\mu_{1}, \sigma_{0}^{2}\right)$. Thus the above probability does not depend on $\tau$ and for computing this probability it can be assumed $\tau=0$. We have,

$$
\begin{aligned}
h\left(k ; \mu_{1}\right)= & P_{\mu_{1}}\left(T^{\star}=k\right)=P_{\mu_{1}}\left(\left\{C_{1}^{+}<h^{+}, C_{1}^{-}<h^{-}\right\} \cap \cdots\right. \\
& \left.\cap\left\{C_{k-1}^{+}<h^{+}, C_{k-1}^{-}<h^{-}\right\} \cap\left\{C_{k}^{+}>h^{+} \text {or } C_{k}^{-}>h^{-}\right\}\right),
\end{aligned}
$$

and the distribution of $T$ is,

$$
\begin{aligned}
g\left(t ; \tau, \mu_{1}\right) & =P_{\mu_{1}}(T=t)=P_{\mu_{1}}\left(T^{\star}+\tau=t\right)=P_{\mu_{1}}\left(T^{\star}=t-\tau\right) \\
& =h\left(t-\tau ; \mu_{1}\right) .
\end{aligned}
$$

\subsection{Approximation of the Distribution of $T$}

Although computing the function $h\left(\cdot ; \mu_{1}\right)$ for every fixed $\mu_{1}$ (and consequently the function $\left.g\left(\cdot ; \tau, \mu_{1}\right)\right)$ is difficult to do, it can be approximated with arbitrary accuracy by using Monte Carlo simulations. To approximate the distribution of $T$, first of all, the following theorem is needed.

Theorem 1. Let $T^{\star}=T-\tau$ be the difference between the signal time $T$ and the change point $\tau$, then as $\Delta \mu=\mu_{1}-\mu_{0}$ approaches plus or minus infinity, the distribution of $T^{\star}$ approaches a degenerate distribution at 1. 


\section{Proof.}

$$
\begin{aligned}
\lim _{\Delta \mu \rightarrow \pm \infty} h\left(1 ; \mu_{1}\right) & =\lim _{\Delta \mu \rightarrow \pm \infty} P_{\mu_{1}}\left(T^{\star}=1\right)=\lim _{\Delta \mu \rightarrow \pm \infty} P_{\mu_{1}}(T-\tau=1) \\
& =\lim _{\Delta \mu \rightarrow \pm \infty} P_{\mu_{1}}(T=\tau+1) \\
& =\lim _{\Delta \mu \rightarrow \pm \infty} P_{\mu_{1}}\left(C_{\tau+1}^{+}>h^{+} \text {or } C_{\tau+1}^{-}>h^{-}\right) \\
& =\lim _{\Delta \mu \rightarrow \pm \infty} P_{\mu_{1}}\left(C_{\tau+1}^{+}>h^{+}\right)+\lim _{\Delta \mu \rightarrow \pm \infty} P_{\mu_{1}}\left(C_{\tau+1}^{-}>h^{-}\right)
\end{aligned}
$$

But we have,

$$
\begin{aligned}
\lim _{\Delta \mu \rightarrow+\infty} P_{\mu_{1}}\left(C_{\tau+1}^{+}>h^{+}\right)= & 1-\lim _{\Delta \mu \rightarrow+\infty} P_{\mu_{1}}\left(C_{\tau+1}^{+}<h^{+}\right) \\
= & 1-\lim _{\Delta \mu \rightarrow+\infty} P_{\mu_{1}}\left(Z_{\tau+1}-k+C_{\tau}^{+}<h^{+}\right) \\
= & 1-\lim _{\Delta \mu \rightarrow+\infty} P\left(\frac{\bar{X}_{\tau+1}-\mu_{1}}{\sigma_{0} / \sqrt{n}}+\frac{\mu_{1}-\mu_{0}}{\sigma_{0} / \sqrt{n}}-k\right. \\
& \left.+C_{\tau}^{+}<h^{+}\right) \\
\geqslant & 1-\lim _{\Delta \mu \rightarrow+\infty} P\left(\frac{\bar{X}_{\tau+1}-\mu_{1}}{\sigma_{0} / \sqrt{n}}+\frac{\Delta \mu}{\sigma_{0} / \sqrt{n}}-k<h^{+}\right) \\
= & 1-\lim _{\Delta \mu \rightarrow+\infty} \Phi\left(-\frac{\Delta \mu \sqrt{n}}{\sigma_{0}}+h^{+}+k\right)=1 .
\end{aligned}
$$

Therefore $\lim _{\Delta \mu \rightarrow+\infty} P_{\mu_{1}}\left(C_{\tau+1}^{+}>h^{+}\right)=1$. Similarly, it is easy to show that $\lim _{\Delta \mu \rightarrow-\infty} P_{\mu_{1}}\left(C_{\tau+1}^{-}>h^{-}\right)=1$, thus, $\lim _{\Delta \mu \rightarrow \pm \infty} h\left(1 ; \mu_{1}\right)=1$.

In the discussion that follows it is assumed that the positive real number $M$ is large enough so that, $h\left(1 ; \mu_{0}+M \sigma_{0}\right) \approx 1$.

Let the interval $\left(\mu_{0}, \mu_{0}+M \sigma_{0}\right)$ be split into congruent subintervals $\left(\mu_{1}^{(i-1)}, \mu_{1}^{(i)}\right)$, $i=1,2, \ldots, N$ with length $\frac{M \sigma_{0}}{N}, \mu_{1}^{(0)}=\mu_{0}$ and $\mu_{1}^{(N)}=\mu_{0}+M \sigma_{0}$. Assume $p_{i k}=h\left(k \mid \mu_{1}^{(i)}\right)$ is specified for $i=0,1, \ldots, N$. Then for each $\mu_{1}^{(i-1)}<\mu_{1}<$ $\mu_{1}^{(i)}$ we approximate $h\left(k \mid \mu_{1}\right)$, the distribution of $T^{\star}$, by a line that passes through two points $\left(\mu_{1}^{(i-1)}, p_{i-1 k}\right)$ and $\left(\mu_{1}^{(i)}, p_{i k}\right)$. Thus, using the symmetry of the normal distribution, the distribution of $T^{\star}$ can be approximated as 
below:

$$
\tilde{h}\left(k ; \mu_{1}\right)=\left\{\begin{array}{lr}
p_{i-1 k}+m_{i \tau}\left(\mu_{1}-\mu_{1}^{(i-1)}\right), & \text { if } \mu_{1}^{(i-1)}<\mu_{1}<\mu_{1}^{(i)} \\
& \text { for } i=1,2, \ldots, N \\
\tilde{h}\left(k \mid 2 \mu_{0}-\mu_{1}\right), & \mu_{0}-M \sigma_{0} \leqslant \mu_{1}<\mu_{0} \\
I_{\{1\}}(k), & \left|\mu_{1}-\mu_{0}\right| \geqslant M \sigma_{0}
\end{array}\right.
$$

where,

$m_{i k}=\frac{p_{i k}-p_{i-1 k}}{\mu_{1}^{(i)}-\mu_{1}^{(i-1)}}=\frac{N\left(p_{i k}-p_{i-1 k}\right)}{M \sigma_{0}}, I_{\{1\}}(k)=\left\{\begin{array}{rr}1 & \text { if } k=1 \\ 0 & \text { o.w }\end{array}\right.$ for $k=1, \ldots, T$.

Using (1) and (2) $g\left(t ; \tau, \mu_{1}\right)$ can be approximated by,

$$
\tilde{g}\left(t ; \tau, \mu_{1}\right)=\tilde{h}\left(t-\tau ; \mu_{1}\right), \quad t=\tau+1, \cdots .
$$

\section{Estimating the Change Point}

Assuming $\mathbf{x}_{\mathbf{i}}=\left(x_{i 1}, \ldots, x_{i n}\right), \quad i=1, \ldots, T$, the joint distribution of the observations, i.e. $\mathbf{x}_{i}$ s given $T$, is

$$
\begin{aligned}
f\left(\mathbf{x}_{1}, \ldots, \mathbf{x}_{T} \mid T, \tau, \mu_{1}\right)= & \prod_{i=1}^{\tau} \prod_{j=1}^{n} f\left(x_{i j}\right) \prod_{i=\tau+1}^{T} \prod_{j=1}^{n} f\left(x_{i j} \mid \mu_{1}\right) \\
= & \left(2 \pi \sigma_{0}^{2}\right)^{-\frac{n t}{2}} \exp \left\{-\frac{n}{2 \sigma_{0}^{2}}\left(\sum_{i=1}^{t} \overline{x_{i}^{2}}-2 \mu_{0} \sum_{i=1}^{\tau} \bar{x}_{i}+\tau \mu_{0}^{2}\right.\right. \\
& \left.\left.-(t-\tau) \overline{\bar{x}}_{\tau}^{2}+(t-\tau)\left(\mu_{1}-\overline{\bar{x}}_{\tau}\right)^{2}\right)\right\}
\end{aligned}
$$

where in the last expression $\overline{\bar{x}}_{\tau}=\frac{1}{t-\tau} \sum_{i=\tau+1}^{t} \bar{x}_{i}$. Throughout the paper, if $\tau=0$, the values of the product $\prod_{i=1}^{\tau}$ and the sum $\sum_{i=1}^{\tau}$ are defined as 1 and 0 , respectively. 


\subsection{Conditional MLE of the Change Point}

Although the signal time $T$ is a random variable, in most of the previous papers it is assumed that $T$ is fixed and a conditional likelihood function given $T$ is used. The MLE of $\tau$ is derived as below,

$$
\hat{\tau}=\arg \max _{\tau}\left\{l^{\star}\left(\tau, \hat{\mu}_{1}(\tau)\right)\right\},
$$

where $l^{\star}\left(\tau, \mu_{1}\right)=\log L^{\star}\left(\tau, \mu_{1}\right)=\log f\left(\mathbf{x}_{1}, \ldots, \mathbf{x}_{T} \mid T, \tau, \mu_{1}\right)$ and $\hat{\mu}_{1}=\frac{1}{n(T-\tau)} \sum_{i=\tau+1}^{T} \sum_{j=1}^{n} x_{i j}$ (For details see Samuel and Pignatiello, 1998).

\subsection{Unconditional MLE of the Change Point}

In practice the signal time $T$ is a random variable, which is observed (with the observed value t, which is fixed) as a part of the observations, and contains some information about the unknown parameter $\tau$ and $\mu$. Thus, its distribution should be contained in the likelihood function. The likelihood function can be rewritten as below,

$$
L\left(\tau, \mu_{1}\right)=f\left(\mathbf{x}_{1}, \ldots, \mathbf{x}_{t} \mid t, \tau, \mu_{1}\right) g\left(t ; \tau, \mu_{1}\right) .
$$

To find the MLE of the parameters $\tau$ and $\mu_{1}$ it suffices to maximize $L\left(\tau, \mu_{1}\right)$ or equivalently maximize $l\left(\tau, \mu_{1}\right)$, the logarithm of the likelihood function, with respect to these parameters. The logarithm of the likelihood function (apart from a constant) is

$$
\begin{aligned}
l\left(\tau, \mu_{1}\right)= & \log L\left(\tau, \mu_{1} \mid \mathbf{x}\right) \propto-\frac{n}{2 \sigma_{0}^{2}}\left\{-2 \mu_{0} \sum_{i=1}^{\tau} \bar{x}_{i}+\tau \mu_{0}^{2}-(t-\tau) \overline{\bar{x}}_{\tau}^{2}\right. \\
& \left.+(t-\tau)\left(\mu_{1}-\overline{\bar{x}}_{\tau}\right)^{2}\right\}+\log g\left(t ; \tau, \mu_{1}\right)
\end{aligned}
$$

we note that there are two unknown parameters in the log-likelihood function: $\tau$ and $\mu_{1}$. For each fixed $\tau, l\left(\tau, \mu_{1}\right)$ can be maximized numerically as a function of $\mu_{1}$, let $\hat{\mu}_{1}(\tau)$ be its maximizer. Substituting this into equation 
(3), we get

$$
\begin{aligned}
l\left(\tau, \hat{\mu}_{1}(\tau)\right)= & -\frac{n}{2 \sigma_{0}^{2}}\left\{-2 \mu_{0} \sum_{i=1}^{\tau} \bar{x}_{i}+\tau \mu_{0}^{2}-(t-\tau) \overline{\bar{x}}_{\tau}^{2}\right. \\
& \left.+(t-\tau)\left(\hat{\mu}_{1}(\tau)-\overline{\bar{x}}_{\tau}\right)^{2}\right\}+g\left(t ; \tau, \hat{\mu}_{1}(\tau)\right) .
\end{aligned}
$$

It then follows that the value of $\tau$ that maximizes the log-likelihood function is,

$$
\hat{\tau}=\arg \max _{\tau}\left\{l\left(\tau, \hat{\mu}_{1}(\tau)\right)\right\} .
$$

That is, $\hat{\tau}$ is the value of $\tau$ in the range $\tau=0,1, \ldots, T-1$ which maximizes $l\left(\tau, \hat{\mu}_{1}(\tau)\right)$. Due to complicated nature of this function, it has to be maximized in $\tau$ numerically, as we show in Section 5. Note that in practice, to determine the likelihood function, probability mass function of $T$, i.e $g\left(t ; \tau, \mu_{1}\right)$ is needed which is not easy to compute and it has to be replaced by its approximation $\tilde{g}\left(t ; \tau, \mu_{1}\right)$.

\section{Finding the True Change Point}

The main goal of estimating the change point is to find the true time at which the disturbances are introduced into the process (or the change point). In most of the previous studies, the evaluation of the performance of the estimators of the change point is based on the direct distance between the estimator $\hat{\tau}$ and the true change point $\tau$, i.e $|\hat{\tau}-\tau|$. In these studies some criteria like $M S E=E\left(|\hat{\tau}-\tau|^{2}\right)$ or $P(|\hat{\tau}-\tau|>i), i \in N$ are used, both of them are based on the direct distance between $\tau$ and $\hat{\tau}$. In the following example it is shown that it is possible a closer estimator has a poorer performance.

Example 1. Suppose that at time $T=104$ the control chart triggers a signal and the true change point is at time point $\tau+1=100$. If we have two estimates $\hat{\tau}_{1}+1=97$ and $\hat{\tau}_{2}+1=104$. Although $\hat{\tau}_{1}+1$ is more close to $\tau+1=100$ than $\hat{\tau}_{2}+1$, but starting from $\hat{\tau}_{1}+1=97$, we may evaluate the process at points $97,98,96,99,95,100$ or $97,96,98,95,99,94,100$ to find the true change points. In other words in average 6.5 points should be checked to get the true change point $\tau+1=100$. While starting from $\hat{\tau}_{2}+1=104$, we may evaluate the time points through the path $104,103,102,101,100$ which 
has 5 points. It can easily be shown that if $\tilde{\tau}$ is an estimate of $\tau$, then the number of points in time which should be examined to find the main causes of the out-of-control signal at time $\tau+1$ is given as the loss function,

$$
L(\tilde{\tau}, \tau)=\left\{\begin{array}{lr}
\tau+1, & \tilde{\tau} \leqslant \frac{\tau-1}{2} \\
2|\tilde{\tau}-\tau|+0.5\left(1+I_{\{\tilde{\tau}\}}(\tau)\right), & \frac{\tau-1}{2}<\tilde{\tau} \leqslant \frac{T-1+\tau}{2} \\
T-\tau, & \tilde{\tau}>\frac{T-1+\tau}{2}
\end{array}\right.
$$

where $I(\cdot)$ is the indicator function.

After estimating the change point by likelihood method, starting from the estimated change point, we should initiate a search to find the true change point. Two methods can be proposed and compared using a trivial loss function which is just the number of points in time which should be examined until the true change point is identified. The first method is called Chronologically-Ordered Method (COM) is as follows. Starting from the estimated change point, the points in time are examined in order of their nearness to the estimated change point. If two points are at the same distance from the estimated change point, they are examined in a random order. In this case, the loss function (4) is exactly the average number of points in time which should be examined to find the true change point. The second method we refer to as Likelihood-ordered Method (LOM) in which starting from the estimated change point, the points in time are examined in order of their likelihood. That is, a point which has a larger likelihood has more priority to be examined as a possible change point. In this case, the loss function is,

$$
L(\hat{\tau}, \tau)=\sum_{i=1}^{T} i I\left(t_{(i)}=\tau\right),
$$

where $t_{(1)}, t_{(2)}, \ldots, t_{(T)}$ are the points $0,1, \ldots, T-1$, decreasingly ordered by their likelihoods and $I(\cdot)$ is the indicator function, note that $\hat{\tau}=t_{(1)}$. In the next section, based on the method used for finding true change point, the loss functions (4) and (5) are applied to compute the risk functions of the methods of finding the true change point. 


\section{Comparison of the Methods}

In this section each one of the aforementioned two methods of finding true change point, i.e. COM and LOM, is applied to both conditional and unconditional MLE of the change point. The four combination of estimatingfinding methods are called, Conditional COM (CCOM), Unconditional COM (UCOM), Conditional LOM (CLOM) and Unconditional LOM (ULOM). Then they are compared through a series of simulations. Suppose $n=1$, sample observations are randomly generated from standard Normal distribution for subgroups $1,2, \ldots, \tau$. Then, starting with subgroup $\tau+1$, observations were randomly generated from $N(\delta, 1)$ until the CUSUM control chart triggers a signal. For each of the values of $\tau=50,100,200$ and $\delta=0.5,1,1.5,2,2.5,3$ this procedure is repeated a total of 1000 times. For each simulation run, both estimators are computed and each one of two proposed methods of finding true change point are applied to both of these estimators. Then the risk function of each one of four combinations of methods of finding and estimating the change point is computed. The average values of conditional and unconditional MLEs of $\tau$ and the signal time $T$, denoted by $\overline{\hat{\tau}}^{(C M L)}, \overline{\hat{\tau}}^{(U M L)}$ and $\bar{T}$ respectively, as well as the risk functions of CCOM, CLOM, UCOM, ULOM and TM are given in Tables 1-3.

We see that for each fixed $\delta$ which is not too small, for $\tau=50,100,200$ the risk of each one of three methods is almost constant. In addition, based on the risk function (Figures 1-3):

For $\tau=50,100,200$, ULOM outperforms all other methods, for almost all values of $\delta$.

For $\tau=50,100,200$, CLOM outperforms almost all three remaining methods, i.e. CCOM, UCOM and TM for $\delta$ which is not too small.

For $\tau=50$, TM is outperformed by all the other four methods. But as $\tau$ increases it appears that for $\tau=100,200$, TM outperforms CCOM for some values of $\delta$.

To evaluate the effect of changing the values of a parameter on the results, we fix all values of the parameters except one which changes. Then the simulations are repeated.

Table 4 shows the results for several different values of $\mu_{0}$ when the other parameters are fixed. As it is seen clearly, ULOM outperforms the other methods. In addition, CLOM outperforms CCOM.

Tables 5-7 show the same results for different values of $\sigma, n$ and small values of $\tau$, respectively when other parameters are fixed.

J. Statist. Res. Iran 15 (2018): 99-117 
Table 1. The mean of the signal time $T$ and MLE of the change point along with the risk function of each of three methods, i.e. TM, COM and LOM, and their standard deviations for different values of $\delta$ (True change point $\tau+1=51$ and subgroup size $n=1$ ).

\begin{tabular}{ccccccc}
\hline \hline$\delta$ & 0.5 & 1 & 1.5 & 2 & 2.5 & 3 \\
\hline $\bar{T}$ & 82.95 & 59.40 & 55.04 & 53.51 & 52.72 & 52.29 \\
& $(0.93)$ & $(0.18)$ & $(0.07)$ & $(0.04)$ & $(0.03)$ & $(0.02)$ \\
\hline$\overline{\hat{\tau}}^{(C M L)}$ & 57.21 & 49.25 & 48.99 & 49.38 & 49.59 & 49.67 \\
& $(0.60)$ & $(0.27)$ & $(0.18)$ & $(0.13)$ & $(0.09)$ & $(0.06)$ \\
\hline$\overline{\hat{\tau}}^{(U M L)}$ & 62.91 & 51.06 & 49.95 & 49.83 & 49.81 & 49.74 \\
& $(0.69)$ & $(0.21)$ & $(0.12)$ & $(0.07)$ & $(0.05)$ & $(0.04)$ \\
\hline$R_{T M}$ & 32.95 & 9.39 & 5.04 & 3.51 & 2.72 & 2.29 \\
& $(0.93)$ & $(0.18)$ & $(0.07)$ & $(0.04)$ & $(0.03)$ & $(0.02)$ \\
\hline$R_{C O M}^{C}$ & 20.28 & 8.11 & 4.39 & 2.98 & 2.19 & 1.81 \\
& $(0.61)$ & $(0.34)$ & $(0.24)$ & $(0.18)$ & $(0.13)$ & $(0.11)$ \\
\hline$R_{L O M}^{C}$ & 15.65 & 6.89 & 3.5 & 2.71 & 1.75 & 1.56 \\
& $(0.53)$ & $(0.30)$ & $(0.20)$ & $(0.16)$ & $(0.09)$ & $(0.08)$ \\
\hline$R_{C O M}^{U}$ & 23.04 & 7.18 & 3.52 & 2.5 & 1.86 & 1.68 \\
& $(0.68)$ & $(0.26)$ & $(0.16)$ & $(0.11)$ & $(0.08)$ & $(0.08)$ \\
\hline$R_{L O M}^{U}$ & 16.54 & 5.82 & 3.06 & 2.43 & 1.69 & 1.57 \\
& $(0.54)$ & $(0.23)$ & $(0.17)$ & $(0.15)$ & $(0.09)$ & $(0.09)$ \\
\hline
\end{tabular}

Table 2. The mean of the signal time $T$ and MLE of the change point along with the risk function of each of three methods, i.e. TM, COM and LOM, and their standard deviations for different values of $\delta$ (True change point $\tau+1=101$ and subgroup size $n=1$ ).

\begin{tabular}{ccccccc}
\hline \hline$\delta$ & 0.5 & 1 & 1.5 & 2 & 2.5 & 3 \\
\hline $\bar{T}$ & 132.69 & 109.09 & 105.01 & 103.52 & 102.67 & 102.27 \\
& $(0.84)$ & $(0.18)$ & $(0.07)$ & $(0.04)$ & $(0.03)$ & $(0.02)$ \\
\hline$\overline{\hat{\tau}}^{(C M L)}$ & 104.81 & 98.45 & 98.51 & 99.10 & 99.37 & 99.58 \\
& $(0.7)$ & $(0.37)$ & $(0.29)$ & $(0.20)$ & $(0.14)$ & $(0.10)$ \\
\hline$\overline{\hat{\tau}}^{(U M L)}$ & 112.29 & 100.85 & 99.80 & 99.68 & 99.67 & 99.72 \\
& $(0.72)$ & $(0.24)$ & $(0.18)$ & $(0.13)$ & $(0.06)$ & $(0.06)$ \\
\hline$R_{T M}$ & 32.7 & 9.09 & 5.01 & 3.52 & 2.67 & 2.27 \\
& $(0.84)$ & $(0.18)$ & $(0.07)$ & $(0.04)$ & $(0.03)$ & $(0.02)$ \\
\hline$R_{C O M}^{C}$ & 22.76 & 9.03 & 5.69 & 3.55 & 2.55 & 1.97 \\
& $(0.75)$ & $(0.53)$ & $(0.40)$ & $(0.31)$ & $(0.21)$ & $(0.16)$ \\
\hline$R_{L O M}^{C}$ & 18.59 & 6.72 & 4.03 & 2.56 & 2.07 & 1.61 \\
& $(0.70)$ & $(0.39)$ & $(0.26)$ & $(0.17)$ & $(0.16)$ & $(0.10)$ \\
\hline$R_{C O M}^{U}$ & 24.31 & 7.03 & 3.98 & 2.66 & 2.11 & 1.75 \\
& $(0.73)$ & $(0.35)$ & $(0.23)$ & $(0.20)$ & $(0.12)$ & $(0.11)$ \\
\hline$R_{L O M}^{U}$ & 18.29 & 5.40 & 3.48 & 2.28 & 1.88 & 1.58 \\
& $(0.64)$ & $(0.28)$ & $(0.24)$ & $(0.15)$ & $(0.14)$ & $(0.11)$ \\
\hline & & & & & & \\
\hline
\end{tabular}


Table 3. The mean of the signal time $\mathrm{T}$ and MLE of the change point along with the risk function of each of three methods, i.e. TM, COM and LOM, and their standard deviations for different values of $\delta$ (True change point $\tau+1=201$ and subgroup size $n=1$ ).

\begin{tabular}{ccccccc}
\hline \hline$\delta$ & 0.5 & 1 & 1.5 & 2 & 2.5 & 3 \\
\hline $\bar{T}$ & 231.79 & 208.74 & 204.99 & 203.50 & 202.73 & 202.31 \\
& $(0.88)$ & $(0.16)$ & $(0.07)$ & $(0.04)$ & $(0.03)$ & $(0.02)$ \\
\hline$\overline{\hat{\tau}}^{(C M L)}$ & 202.13 & 197.31 & 198.31 & 199.39 & 199.10 & 199.51 \\
& $(0.90)$ & $(0.59)$ & $(0.35)$ & $(0.13)$ & $(0.29)$ & $(0.20)$ \\
\hline$\overline{\hat{\tau}}^{(U M L)}$ & 210.75 & 200.11 & 199.62 & 199.81 & 199.74 & 199.81 \\
& $(0.82)$ & $(0.40)$ & $(0.18)$ & $(0.07)$ & $(0.06)$ & $(0.04)$ \\
\hline$R_{T M}$ & 31.79 & 8.74 & 4.99 & 3.50 & 2.73 & 2.31 \\
& $(0.88)$ & $(0.16)$ & $(0.07)$ & $(0.04)$ & $(0.03)$ & $(0.02)$ \\
\hline$R_{C O M}^{C}$ & 25.28 & 11.02 & 6.18 & 3.15 & 2.87 & 2.03 \\
& $(1.07)$ & $(0.85)$ & $(0.6)$ & $(0.24)$ & $(0.33)$ & $(0.23)$ \\
\hline$R_{L O M}^{C}$ & 22.75 & 8.54 & 5.08 & 2.78 & 2.03 & 1.76 \\
& $(1.07)$ & $(0.62)$ & $(0.50)$ & $(0.27)$ & $(0.16)$ & $(0.15)$ \\
\hline$R_{C O M}^{U}$ & 24.80 & 7.75 & 4.23 & 2.47 & 2.01 & 1.64 \\
& $(0.87)$ & $(0.52)$ & $(0.34)$ & $(0.11)$ & $(0.10)$ & $(0.08)$ \\
\hline$R_{L O M}^{U}$ & 20.41 & 6.49 & 4.03 & 2.29 & 2.04 & 1.68 \\
& $(0.88)$ & $(0.47)$ & $(0.43)$ & $(0.23)$ & $(0.25)$ & $(0.11)$ \\
\hline & & & & & &
\end{tabular}

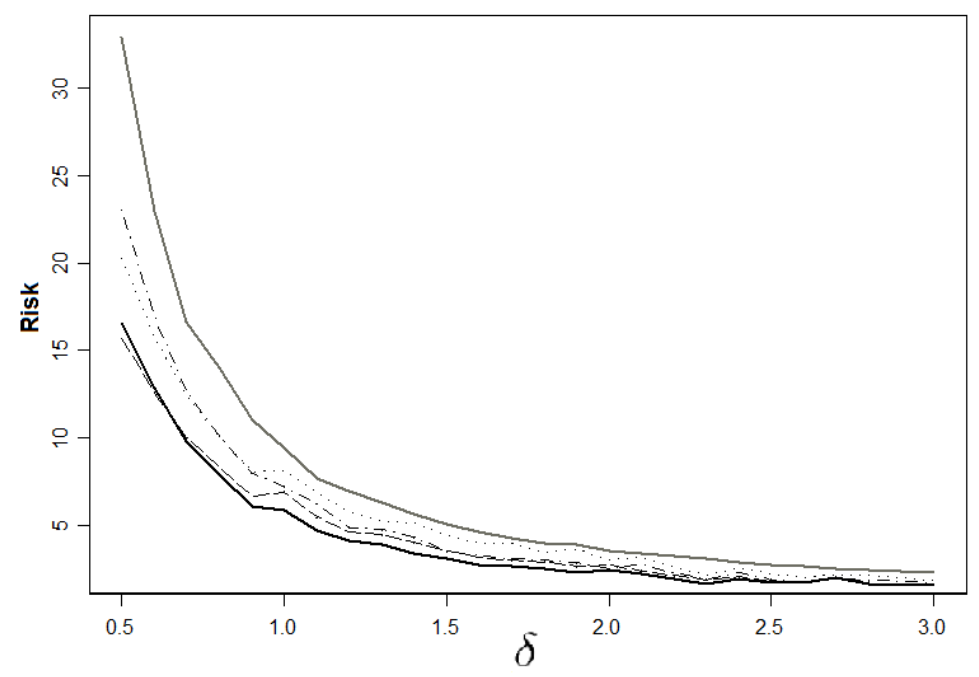

Figure 1. The risk functions of ULOM (solid curve), UCOM (broken curve), CLOM (dotdashed curve), CCOM (dotted curve) and TM (light solid) (True change point $\tau+1=51$ and subgroup size $n=1)$. 


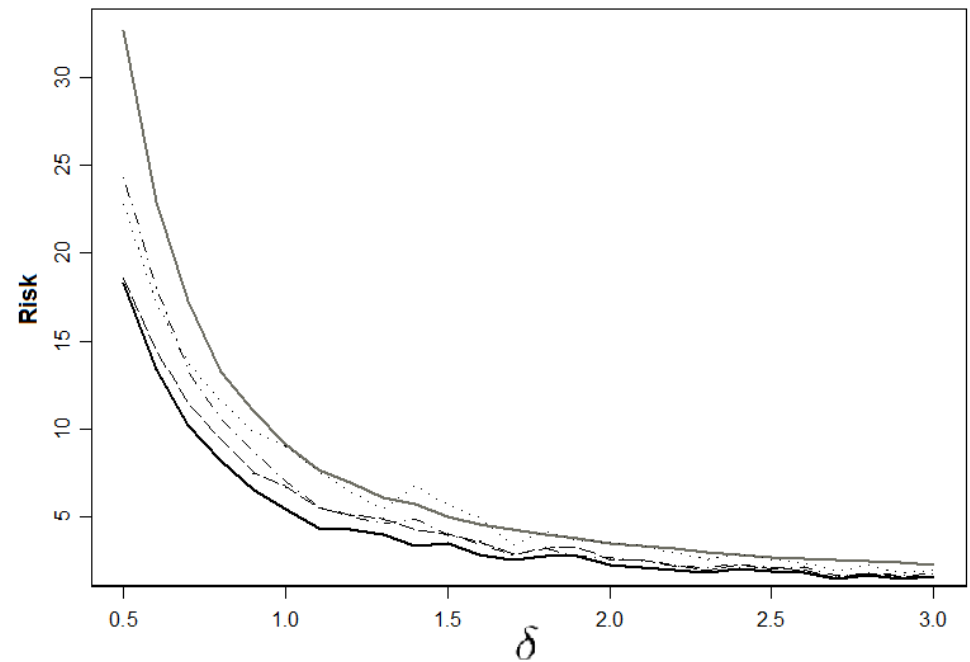

Figure 2. The risk functions of ULOM (solid curve), UCOM (broken curve), CLOM (dotdashed curve), CCOM (dotted curve) and TM (light solid) (True change point $\tau+1=101$ and subgroup size $n=1)$.

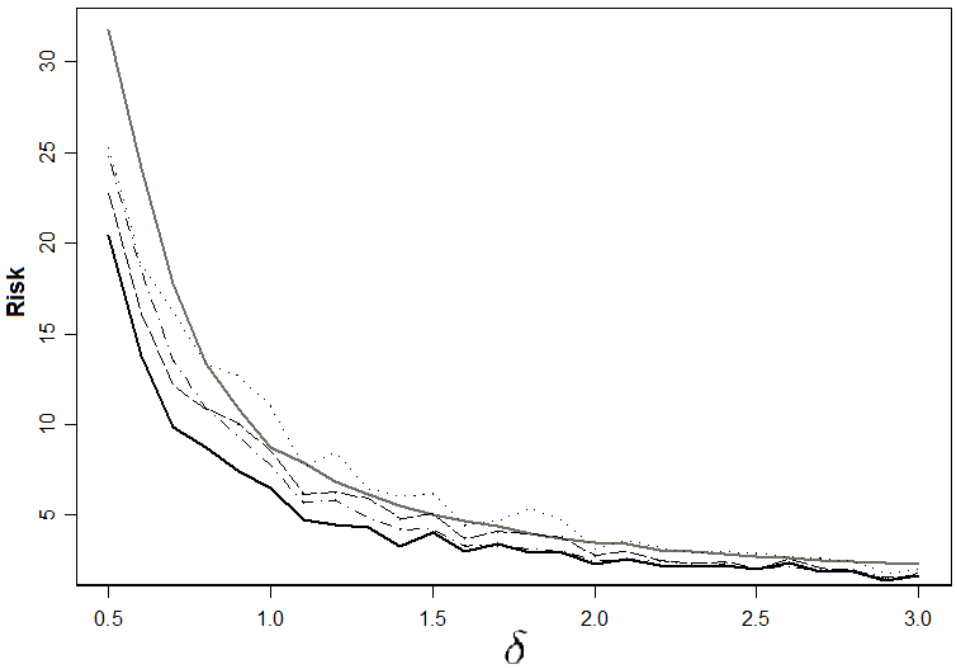

Figure 3. The risk functions of ULOM (solid curve), UCOM (broken curve), CLOM (dotdashed curve), CCOM (dotted curve) and TM (light solid) (True change point $\tau+1=201$ and subgroup size $n=1$ ). 
Table 4. The mean of the signal time $T$ and MLE of the change point along with the risk function of each of three methods, i.e. TM, COM and LOM, and their standard deviations for $n=1, \tau+1=21, \delta=1, \sigma=1$ and different values of $\mu_{0}$.

\begin{tabular}{ccccccc}
\hline \hline$\mu_{0}$ & 0.5 & 1 & 1.5 & 2 & 2.5 & 3 \\
\hline $\bar{T}$ & 29.45 & 30.32 & 28.98 & 30.28 & 28.64 & 28.49 \\
& $(5.35)$ & $(5.54)$ & $(5.60)$ & $(6.14)$ & $(7.23)$ & $(5.36)$ \\
\hline$\overline{\hat{\tau}}^{(C M L)}$ & 20.47 & 21.15 & 19.30 & 20.53 & 20.24 & 20.86 \\
& $(6.32)$ & $(5.18)$ & $(6.07)$ & $(5.96)$ & $(4.38)$ & $(4.25)$ \\
\hline$\overline{\hat{\tau}}^{(U M L)}$ & 22.24 & 22.52 & 21.19 & 22.28 & 20.82 & 21.76 \\
& $(5.01)$ & $(4.91)$ & $(5.46)$ & $(6.32)$ & $(5.30)$ & $(4.81)$ \\
\hline$R_{T M}$ & 9.45 & 10.32 & 8.98 & 10.28 & 8.64 & 8.49 \\
& $(5.35)$ & $(5.54)$ & $(5.60)$ & $(6.14)$ & $(7.23)$ & $(5.36)$ \\
\hline$R_{C O M}^{C}$ & 7.13 & 5.84 & 6.18 & 6.26 & 5.56 & 5.72 \\
& $(6.58)$ & $(5.55)$ & $(6.05)$ & $(6.37)$ & $(5.03)$ & $(5.18)$ \\
\hline$R_{L O M}^{C}$ & 5.17 & 4.6 & 4.74 & 5.43 & 5.16 & 4.57 \\
& $(4.51)$ & $(4.50)$ & $(4.24)$ & $(5.81)$ & $(5.15)$ & $(4.48)$ \\
\hline$R_{C O M}^{U}$ & 6.83 & 6.22 & 5.98 & 6.93 & 6.07 & 5.84 \\
& $(5.75)$ & $(5.72)$ & $(5.5)$ & $(6.83)$ & $(5.68)$ & $(5.31)$ \\
\hline$R_{L O M}^{U}$ & 4.84 & 4.71 & 4.65 & 5.15 & 5.17 & 4.44 \\
& $(4.13)$ & $(4.56)$ & $(4.14)$ & $(5.37)$ & $(4.86)$ & $(4.37)$ \\
\hline
\end{tabular}

Table 5. The mean of the signal time $T$ and MLE of the change point along with the risk function of each of three methods, i.e. TM, COM and LOM, and their standard deviations for $n=1, \mu_{0}=0, \tau+1=21, \delta=1$, and different values of $\sigma$.

\begin{tabular}{cccccc}
\hline \hline$\sigma$ & 1 & 1.5 & 2 & 2.5 & 3 \\
\hline $\bar{T}$ & 28.89 & 29.69 & 28.39 & 29.85 & 29.16 \\
& $(5.10)$ & $(5.49)$ & $(4.92)$ & $(5.43)$ & $(5.67)$ \\
\hline$\overline{\hat{\tau}}^{(C M L)}$ & 19.78 & 20.02 & 19.99 & 20.85 & 19.63 \\
& $(6.50)$ & $(4.98)$ & $(5.15)$ & $(6.26)$ & $(4.66)$ \\
\hline$\overline{\hat{\tau}}^{(U M L)}$ & 21.48 & 20.94 & 20.26 & 20.94 & 20.63 \\
& $(6.23)$ & $(3.89)$ & $(4.64)$ & $(6.30)$ & $(4.66)$ \\
\hline$R_{T M}$ & 8.89 & 9.69 & 8.39 & 9.85 & 9.16 \\
& $(5.10)$ & $(5.49)$ & $(4.92)$ & $(5.43)$ & $(5.67)$ \\
\hline$R_{C O M}^{C}$ & 6.70 & 5.26 & 5.54 & 7.37 & 5.82 \\
& $(6.43)$ & $(5.44)$ & $(5.47)$ & $(6.18)$ & $(5.52)$ \\
\hline$R_{L O M}^{C}$ & 4.45 & 3.50 & 5.08 & 5.67 & 4.69 \\
& $(5.13)$ & $(3.21)$ & $(5.84)$ & $(4.83)$ & $(4.59)$ \\
\hline$R_{C O M}^{U}$ & 6.92 & 4.64 & 5.41 & 7.37 & 5.82 \\
& $(6.20)$ & $(4.79)$ & $(5.23)$ & $(6.22)$ & $(5.52)$ \\
\hline$R_{L O M}^{U}$ & 4.26 & 3.39 & 5.08 & 5.66 & 4.68 \\
& $(4.64)$ & $(2.97)$ & $(5.76)$ & $(4.77)$ & $(4.49)$ \\
\hline & & & & &
\end{tabular}


Table 6 . The mean of the signal time $T$ and MLE of the change point along with the risk function of each of three methods, i.e. TM, COM and LOM, and their standard deviations for $\mu_{0}=0, \tau+1=21, \delta=1, \sigma=1$, and different values of $n$.

\begin{tabular}{ccccc}
\hline \hline$n$ & 2 & 4 & 8 & 10 \\
\hline $\bar{T}$ & 28.97 & 28.79 & 29.43 & 28.90 \\
& $(5.11)$ & $(6.35)$ & $(4.94)$ & $(5.30)$ \\
\hline$\overline{\hat{\tau}}^{(C M L)}$ & 19.09 & 20.41 & 21.10 & 20.28 \\
& $(6.00)$ & $(5.73)$ & $(4.78)$ & $(5.16)$ \\
\hline$\overline{\hat{\tau}}^{(U M L)}$ & 20.71 & 21.57 & 22.09 & 21.43 \\
& $(4.66)$ & $(5.83)$ & $(4.82)$ & $(5.50)$ \\
\hline$R_{T M}$ & 8.97 & 8.79 & 9.43 & 8.90 \\
& $(5.11)$ & $(6.35)$ & $(4.94)$ & $(5.30)$ \\
\hline$R_{C O M}^{C}$ & 6.44 & 5.92 & 5.95 & 6.01 \\
& $(6.57)$ & $(6.25)$ & $(5.72)$ & $(5.54)$ \\
\hline$R_{L O M}^{C}$ & 5.21 & 4.91 & 4.05 & 4.48 \\
& $(5.43)$ & $(4.98)$ & $(3.29)$ & $(4.35)$ \\
\hline$R_{C O M}^{U}$ & 5.55 & 6.11 & 5.91 & 6.22 \\
& $(5.33)$ & $(6.13)$ & $(5.68)$ & $(5.67)$ \\
\hline$R_{L O M}^{U}$ & 4.94 & 4.84 & 4.07 & 4.42 \\
& $(5.26)$ & $(4.66)$ & $(3.44)$ & $(4.16)$ \\
\hline
\end{tabular}

Table 7. The mean of the signal time $T$ and MLE of the change point along with the risk function of each of three methods, i.e. TM, COM and LOM, and their standard deviations for $n=1, \mu_{0}=0, \delta=1, \sigma=1$, and different values of $\tau$.

\begin{tabular}{ccccc}
\hline \hline$\tau$ & 5 & 10 & 20 & 40 \\
\hline $\bar{T}$ & 14.18 & 17.57 & 27.76 & 48.31 \\
& $(5.68)$ & $(4.88)$ & $(5.54)$ & $(5.10)$ \\
\hline$\overline{\hat{\tau}}^{(C M L)}$ & 5.75 & 9.19 & 20.19 & 39.41 \\
& $(3.69)$ & $(4.06)$ & $(5.19)$ & $(6.70)$ \\
\hline$\overline{\hat{\tau}}^{(U M L)}$ & 4.02 & 10.04 & 20.69 & 41.06 \\
& $(5.02)$ & $(3.97)$ & $(5.01)$ & $(4.91)$ \\
\hline$R_{T M}$ & 10.18 & 8.57 & 8.76 & 9.31 \\
& $(5.68)$ & $(4.88)$ & $(5.54)$ & $(5.10)$ \\
\hline$R_{C O M}^{C}$ & 4.75 & 5.08 & 5.83 & 6.56 \\
& $(4.80)$ & $(4.24)$ & $(6.05)$ & $(7.94)$ \\
\hline$R_{L O M}^{C}$ & 3.64 & 4.15 & 5.14 & 5.30 \\
& $(3.10)$ & $(3.34)$ & $(5.87)$ & $(6.68)$ \\
\hline$R_{C O M}^{U}$ & 5.64 & 5.08 & 5.71 & 6.02 \\
$R_{L O M}^{U}$ & $(6.29)$ & $(4.49)$ & $(5.76)$ & $(5.71)$ \\
\hline & $(3.68)$ & $(3.33)$ & $(5.43)$ & $(6.02)$ \\
\hline
\end{tabular}




\section{Conclusion}

In this study, two MLE estimates for the change point, when implementing a CUSUM control chart, were computed. The first one which is called conditional MLE is the MLE of the change point that is computed from a conditional likelihood given the signal time of the control chart. The second estimate is called unconditional MLE. This second MLE is computed from an unconditional likelihood that contains some extra information of the signal time $T$ about the change point. In addition starting from each one of these MLEs, two methods are proposed to find the true change point, namely LOM and COM.

When these two methods are used for conditional MLE, they were shown as CLOM and CCOM respectively and when they were used for unconditional MLE, they were shown as ULOM and UCOM. By comparing CLOM, CCOM, ULOM and UCOM it was concluded that ULOM outperforms the other methods based on a trivial risk function which is the mean of the number of the points in time that should be examined to find the true change point. In addition, both COM and LOM, when used in an unconditional situation, have better performances. The results hold even when the in control mean, standard deviation of the process and subgroup sample size $n$ change. In this paper, we used the CUSUM control chart to monitor a normal process. There are other types of control charts (for example, EWMA and S control charts) which could be considered and our proposed methods applied.

\section{Acknowledgment}

The authors would like to thank the referees and editors for supplying extremely helpful comments and suggestions.

\section{References}

Cheng, C.S. and Cheng, H.P. (2008). Identifying the Source of Variance Shifts in the Multivariate Process Using Neural Networks and Support Vector Machines. Expert Syst. Appl., 35, 198-206.

Dehghan Monfared, M.E. and Lak, F. (2013). Bayesian Estimation of the Change Point Using $\bar{X}$ Control Chart, Communications in Statistics-Theory and Methods, 42, 1572-1582.

Dehghan Monfared, M.E. and Lak, F. (2016). Bayesian Method for Finding True Change Point when a Control Chart is Used, J. Statist. Res. Iran, 13, 107- 117.

J. Statist. Res. Iran 15 (2018): 99-117 
Dehghan Monfared, M.E. and Lak, F. (2017). Comparing Two MLEs of The Change Point When an $\bar{X}$ Control Chart Is Used, Journal of Statistical Theory and Applications, 16, 209218 .

Dehghan Monfared, M.E. and Meshkani, M.R. (2007). Bayesian Estimation of the Multiple Change Points in a Gamma Process Using $\bar{X}$ Chart, J. Statist. Res. Iran, 4, 203- 216.

Hou, C.D., Shao, Y.E. and Huang, S. (2013). A Combined MLE and Generalized P Chart Approach to Estimate the Change Point of a Multinomial Process, Applied Mathematics \& Information Sciences, 7, 1487-1493.

Montgomery, D.C. (2009). Introduction to Statistical Quality Control, 6th ed., Wiley, New York.

Pignatiello, J.J. and Samuel, T.R. (2001). Estimation of the Change Point of a Normal Process Mean in SPC Applications, Journal of Quality Technology, 33, 82-95.

Samuel, T.R., Pignatiello, J.J. and Calvin, J.A. (1998). Identifying the Time of a Step Change with $\bar{X}$ Control Charts, Quality Engineering, 10, 521-527.

Shao, Y.E. and Hou, C.D. (2004). A Study on Identifying the Time of a Step Change Disturbance with S Control Charts and MLE Method. Journal of the Chinese Institute of Industrial Engineers, 21, 349-357.

Shao, Y.E. and Hou, C.D. (2006). Estimation of the Starting Time of a Step Change Disturbance in a Gamma Process. Journal of the Chinese Institute of Industrial Engineers, 23, 319-327.

Shao, Y.E. and Hou, C.D. (2013a). Fault Identification in Industrial Processes Using an Integrated Approach of Neural Network and Analysis of Variance. Mathematical Problems in Engineering, article ID 516760, 1-7.

Shao, Y.E. and Hou, C.D. (2013b). Change Point Determination for a Multivariate Process Using a Two-stage Hybrid Scheme. Applied Soft Computing, 13, 1520-1527.

Shao, Y.E., Hou, C.D. and Wang, H.J. (2006). Estimation of the Change Point of a Gamma Process by Using the S Control Chart and MLE. Journal of the Chinese Institute of Industrial Engineers, 23, 207-214.

Shao, Y.E. and Hsu, B.S. (2009). Determining the Contributers for a Multivariate SPC Chart Signal Using Artificial Neural Networks and Aupport Vector Machine", Int. J. INNovative Comput. Inform. Cont., 5, 4899-4906. 


\section{Mohammad Esmaeil Dehghan Monfared}

Department of Statistics,

Faculty of Sciences,

Persian Gulf University,

Bushehr, Iran.

email:monfared2@gmail.com

\section{Fazlollah Lak}

Department of Statistics,

Faculty of Sciences,

Persian Gulf University,

Bushehr, Iran.

email:fazlollahlak@gmail.com 
